\title{
Quelques exemples récents d'utilisation de méthodes d'examen ou d'analyse des matériaux du patrimoine culturel mettant en œuvre des rayons $X$
}

\author{
J.-L. Boutaine \\ Centre de Recherche et de Restauration des Musées de France, Laboratoire des Musées \\ de France, 6 rue des Pyramides, 75041 Paris cedex 01, France
}

\begin{abstract}
Résumé : Le Centre de Recherche et de Restauration des Musées de France (C2RMF) a, parmi ses missions, celle de développer et d'appliquer des techniques d'examen, de caractérisation et d'analyse des oeuvres d'art ou du patrimoine culturel, des matériaux constitutifs de ces oeuvres, afin de connaître les mécanismes de leur élaboration, de leur évolution / dégradation et d'établir des recommandations en vue de leur restauration et de leur conservation. Pour cela, il privilégie les méthodes non-destructives, sans contact, sans prélèvement. Parmi ces méthodes, certaines mettent en cuvre les rayons $\mathrm{X}$ : radiographie $\mathrm{X}$, émissiographie, radiographie par électrons secondaires, stratigraphie, diffraction $X$, microscopie électronique à balayage (MEB) et analyse par spectrométrie $X$ associée, analyse par fluorescence $X$, analyse par faisceaux d'ions (AGLAE) : PLXE et fluorescence $X$ secondaire. On sollicite également des laboratoires extérieurs (tomodensitométrie, caractérisation structurale à l'aide du rayonnement synchrotron). Ces méthodes, seules ou associées à d'autres méthodes sont appliquées à des cuvres très diverses et des matériaux de toute nature, de toute origine et de tout âge. On illustrera ces techniques à propos de divers exemples récents d'objets de collections publiques françaises étudiés par le Centre.
\end{abstract}

\section{LE CENTRE DE RECHERCHE ET DE RESTAURATION DES MUSEES DE FRANCE (C2RMF)}

\subsection{Présentation}

C'est un service de la Direction des Musées de France (DMF), du Ministère de la Culture et de la Communication, créé fin 1998 par fusion du Laboratoire (LRMF) et du Service de Restauration (SRMF). Il est situé à Paris et à Versailles. Il comprend quatre départements : Conservation - Restauration, Recherche, Prévention et Documentation - Imagerie Scientifique. Il comprend environ 160 agents permanents.

Le Laboratoire (Département Recherche) compte aujourd'hui 60 agents permanents. C'est une Unité Mixte de Recherche du CNRS (UMR 171). Il se consacre à la recherche, au développement et aux applications de techniques d'examen, de caractérisation et d'analyse des oeuvres d'art ou du patrimoine culturel, des matériaux constitutifs de ces oeuvres, afin de connaître les mécanismes de leur élaboration, de leur évolution / dégradation et d'établir des recommandations en vue de leur restauration et de leur conservation

\subsection{Modes d'intervention}

Le Laboratoire intervient :

- à l'initiative des responsables des collections des musées nationaux ou relevant de collectivités territoriales, des structures gérant l'archéologie, des bibliothèques, des archives... en réalisant des examens ou analyses non destructives, soit pour l'assistance à la restauration, soit pour l'authentification lors de propositions d'acquisition (ex : objets d'art mésoaméricains, africains ou océaniens, pour le futur Musée des Arts et des Civilisations, quai 
Branly), ou encore à la demande des autorités judiciaires (ex : faux, trafic illicite d'oeuvres d'art).

- à l'occasion d'expositions importantes (ex : Georges de la Tour, Un ami de Cézanne et de van Gogh, le docteur Gachet - Grand Palais) lors de rénovation de Musées (Guimet) ou de création de Musées (Musée des Arts et des Civilisations, quai Branly) en effectuant des études approfondies sur des ensembles.

- en menant des actions de recherche propre sur certaines thématiques telles que :

- Physico-chimie des matériaux du patrimoine, transformation de ces matériaux, soit voulues par l'homme, soit subies par l'œuvre (temps, environnement, climat, fonction propre).

- Histoire des techniques : palette des sarcophages égyptiens, élaboration et patine des bronzes et alliages cuivreux archéologiques, technique des céramiques glaçurées.

- Mise au point de nouvelles méthodes d'analyse ou d'examen :

- analyse par faisceau d'ions (dont PIXE)

- numérisation d'images en très haute définition

- analyse par fluorescence X portable

- spectrocolorimétrie portable

\subsection{Principales techniques mises en oeuvre}

- Photographie ; c'est la technique la plus utilisée : photographie classique, photographie sous éclairage en lumière rasante, photographie infrarouge par réflexion $(\lambda=750$ à $900 \mathrm{~nm})$, photographie de la fluorescence sous éclairage ultraviolet $(\lambda=320$ à $400 \mathrm{~nm}$ ), réflectographie infrarouge avec une caméra électronique $(\lambda=1800$ à $2500 \mathrm{~nm})$

- Microscopie optique et électronique (MEB)

- Radiographie

- Numérisation et transmission d'images en très haute définition

- Datation par thermoluminescence et par luminescence stimulée optiquement

- Diffraction X

- Analyse par fluorescence X et par spectrométrie X associée au MEB

- Analyse par faisceaux d'ions (AGLAE) : PIXE, RBS et réactions nucléaires

- Chromatographie en phase gazeuse couplée à la spectrométrie de masse (GC-MS)

- Spectrométrie d'émission atomique (ICP-AES)

- Spectrométrie infrarouge

- Spectrocolorimétrie

\subsection{Matériaux étudiés}

Tous types de matériaux, de toutes provenances, de tous âges. Parmi les plus fréquents :

- roches, gemmes, terres cuites, céramiques, émaux, verres

- bois, papiers, tissus, os, ivoire

- métaux (fer et alliages, cuivre et alliages, or, argent, plomb et alliages)

- couches picturales, supports toile et bois, pigments, huiles, liants, vernis, colles

- matières synthétiques mises en oeuvre au XIX et $\mathrm{XX}^{\mathfrak{e}}$ siècles

- etc...

\subsection{Base NARCISSE}

C'est une base de données Texte + Images, développée dans le cadre de projets de recherche européens, permettant d'accéder à des dossiers d'œuvre, intégrant notices sur les oeuvres, bibliographies, rapports d'étude et images. 
Caractéristiques principales de la base Images : numérisation en très haute définition ( $5000 \mathrm{x}$ 7000 pixels pour un cliché $20 \times 25 \mathrm{~cm}$ ), grandissement maximal X 256, codage de la gamme de gris ou de couleurs sur 24 bits, logiciel permettant d'effectuer le "mosaïcage" des clichés, jusqu'à de très grands formats (plusieurs $\mathrm{m}^{2}$ ) ; -60000 images sont actuellement enregistrées dans la base. Elle est accessible sur le réseau intranet du Ministère de la Culture. Une version réduite est consultable sur le site internet :

\section{http://www.culture.fr/, puis base de données, puis Narcisse}

\subsection{Autres laboratoires et structures analogues}

D'autres structures ont des missions voisines :

En France, citons le LRMH (Laboratoire de Recherche des Monuments Historiques), le CRCDG (Centre de Recherche sur la Conservation des Documents Graphiques), les laboratoires de la BNF, du Musée de la Musique... et vingt et un ateliers régionaux de restauration dont certains ont une capacité de recherche (NANCY, NANTES, GRENOBLE).

On pourra consulter le très intéressant site internet du Ministère de la Culture et de la Communication :

\section{http://www.culture.fr}

et à l'intérieur de ce site les pages consacrées à la documentation et à la recherche, en particulier:

\section{http://www.culture.gouv.fr/culture/conservation/fr/methodes/methodes.htm}

Dans le Monde, citons quelques homologues célèbres : en Italie, Istituto Centrale del Restauro - ROME, Opificio delle Pietre Dure - FLORENCE, en Belgique, Institut Royal du Patrimoine Artistique (IRPA) - BRUXELLES, aux Pays-Bas, Instituut van Collectie Nederland (ICN) AMSTERDAM, en Grande Bretagne, Laboratoires du British Museum, de la National Gallery et du Victoria \& Albert Museum - LONDRES, au Canada, Institut Canadien de Conservation - OTTAWA, aux Etats-Unis, Smithsonian Center for Materials Research and Education Smithsonian Institution - WASHINGTON DC, Getty Conservation Institute - LOS ANGELES ...

Dans un souci de mieux utiliser les compétences existant dans différents laboratoires, d'accroitre les moyens humains et techniques, de partager les connaissances et de travailler en réseau, le Laboratoire s'est engagé dans un Groupement de recherche CNRS intitulé CHIMART regroupant 23 laboratoires français et dans un réseau européen intitulé LABSTECH, dans le cadre du 5 ème PCRD - Ville de demain et patrimoine culturel, animé par l'Université de PEROUSE (11 laboratoires d'Allemagne, Belgique, France, Grande Bretagne, Grèce, Italie et Portugal).

\section{ELEMENTS RELATIFS AUX TECHNIQUES METTANT EN GUVRE LES RAYONS X}

\subsection{Radiographie}

Le Laboratoire met en oeuvre une panoplie de techniques radiographiques conventionnelles et " moins conventionnelles". Rappelons qu'il s'agit d'un examen non destructif, en général par transmission d'un rayonnement ionisant pénétrant. Dans l'objet examiné, peuvent avoir lieu diverses interactions rayonnement ionisant / matière : absorption vraie, diffusion, émission de rayonnement secondaire..., ceci avec des probabilités différentes selon la nature $\mathrm{du}$ rayonnement mis en oeuvre, son énergie et la nature du ou des matériaux constitutifs de l'objet examiné. 


\subsubsection{Radiographie $X$ à basse tension}

Utilisation de générateurs de 15 à $60 \mathrm{kV}$, grande distance source - film, film lent - bonne définition, utilisé sans écran renforçateur, en grand format, jusqu'à $40 \times 150 \mathrm{~cm}$. Technique analogue à la radiographie médicale ou à la radiographie mise en oeuvre dans le contrôle en aéronautique. C'est la technique utilisée quasi systématiquement pour l'examen des peintures de chevalet sur supports toile et bois.

\subsubsection{Radiographie $X \grave{a}$ haute tension}

Utilisation de générateurs jusqu'à $420 \mathrm{kV}$, pour contrôle d'objets divers : statues en pierre, en bronze, mobilier, orfèvrerie, poteries, céramiques, instruments de musique, ustensiles des arts et traditions populaires (très semblable à la radiographie industrielle). En général, film avec écrans renforçateurs métalliques, et plus rarement radioscopie avec amplificateur de brillance.

\subsubsection{Radiographie gamma (en collaboration avec le CEA)}

Utilisation d'appareils de radiographie gamma ( ${ }^{192}$ Ir jusqu'à $300 \mathrm{~mm}$ de pierre ou ${ }^{60} \mathrm{Co}$ jusqu'à $450 \mathrm{~mm}$ de pierre) pour le contrôle de statues de fortes épaisseurs (ex : marbre métopes d'Olympie, gladiateur Borghese, grès - statues khmères d'Angkor).

\subsubsection{Bêtagraphie}

Radiographie par contact de feuilles minces, principalement de papier, à l'aide d'une source plaque émettrice de particules $\beta$, carbone $14\left(\mathrm{E}_{\beta} \max =156 \mathrm{keV}\right)$ en utilisant un film rapide monocouche. La bêtagraphie permet de très bien mettre en évidence la texture du papier (variations locales du grammage), les vergeures, les empreintes des pontuseaux \& surtout les filigranes, en s'affranchissant du texte ou du dessin.

\subsubsection{Emissiographie}

Utilisation de la couche superficielle d'oeuvres planes, comme convertisseur photons X / électrons (épaisseur de couche intéressée de l'ordre de $100 \mu \mathrm{m}$, rendement de conversion variable en fonction du numéro atomique du matériau examiné). Mise en œuvre d'un générateur $\mathrm{X}$ réglé vers $300 \mathrm{kV}$, très filtré (environ $10 \mathrm{~mm} \mathrm{Cu}$ ), film radiographique monocouche disposé contre la couche picturale et coté faisceau incident (technique valable aussi bien pour les peintures de chevalet sur supports toile, que bois, cuivre ou pierre, que pour les émaux cloisonnés ou champlevés)

Le Laboratoire utilise plus rarement le stratigraphie $X$, la neutronographie et l'autoradiographie et la tomographie.

\subsection{Diffraction X}

C'est un outil classique, d'utilisation très fréquente, pour caractériser les structures cristallines (pigments minéraux, roches, constituants des céramiques, métaux et alliages et leurs produits d'altération). La mise en cuvre récente d'un miroir de Göbel permet en incidence rasante l'étude de patines et de surfaces altérées (noircissement d'objets en argent, altération des bulles papales en plomb...).

Pour certaines études (ex. cosmétiques égyptiens à base de composés de plomb), l'emploi du rayonnement synchrotron (LURE - ORSAY ou ESRF - GRENOBLE) est nécessaire étant donné la faible masse d'échantillon disponible (qq. $\mathrm{mg}$ ). 


\subsection{Microscopie à balayage (MEB) et spectrométrie $\mathrm{X}$ associée}

Différents types de microscopes sont utilisés :

- optique métallographique, pour échantillons polis

- optique pétrographique, pour lames minces

- électronique à balayage (MEB)

- électronique à transmission (MET), pour échantillons ultra minces (sous-traité)

L'examen au microscope électronique à balayage, est très utilisé, associé aux dispositifs de microanalyse par spectrométrie $\mathrm{X}$; leurs principales caractéristiques sont :

- analyse sur échantillons (donc prélèvements)

$-\mathrm{Z}>6$ à $8(\mathrm{C}$ à $\mathrm{O})$

- limite pratique de détection qq. $10^{-6}$

- examen microscopique, spot de très petite dimension, de l'ordre de $\varnothing$ qq. nm pour l'image à qq. $\mu \mathrm{m}$ pour l'interaction, en surface, associé à une cartographie

Applications : examen et analyse de la matière picturale dans les coupes stratigraphiques, détermination du profil de teneur de plusieurs constituants dans la couche superficielle corrodée d'un alliage archéologique, analyse des constituants minéraux de terres cuites, poteries, émaux, céramiques, glaçures, verres, roches, pierres précieuses, pigments, bois minéralisés, tissus minéralisés, etc...

\subsection{Analyse par fluorescence X}

Le Laboratoire a choisi de développer un prototype sur la base des spécifications suivantes : - tube à rayons $\mathrm{X}$ léger $(\sim 1 \mathrm{~kg})$, anticathode en $\mathrm{Mo}$, compact, à refroidissement à air, avec fenêtre de sortie peu absorbante $(\sim 200 \mu \mathrm{m} \mathrm{Be})$

- alimentation HT $(60 \mathrm{kV})$ compacte, légère, régulée et pouvant être contrôlée par ordinateur

- détecteur de rayons $\mathrm{X}$ en silicium, refroidissement par effet Peltier, résolution de $150 \mathrm{eV}$ à

$5.9 \mathrm{keV}$, fenêtre peu absorbante $(8 \mu \mathrm{m} \mathrm{Be})$

- montage réduisant l'absorption sur le parcours des photons $X$ dans la région 1-10 keV (pompage ou flux d'hélium)

- analyseur multicanal compact couplé à un calculateur portable

- dispositif de visualisation à l'aide d'une caméra vidéo miniature

L'objectif est de pouvoir effectuer des spectres X permettant l'analyse in situ des éléments majeurs et mineurs entre $\mathrm{Na}$ et $\mathrm{U}$. Cet appareil permettra l'identification sans contact et sans prélèvement des matériaux des objets de musée et d'archéologie. Applications : analyse élémentaire des pigments des peintures, fresques, grottes ornées, sur site

\subsection{Analyse par faisceaux d'ions (AGLAE), PIXE et fluorescence $X$ secondaire}

Le LRMF est le seul laboratoire au monde à disposer d'un accélérateur dédié à l'analyse d'oeuvres d'art ou d'objets du patrimoine culturel par faisceaux d'ions. AGLAE (Accélérateur Grand Louvre pour l'Analyse Elémentaire) est un accélérateur tandem NEC Pelletron $2 \mathrm{MV}$.

\subsubsection{PIXE}

Le principal mode d'analyse utilisé est le mode PIXE (Particle Induced X-ray Emission)

Les principales caractéristiques sont :

- analyse directement sur l'objet, mais limité à la surface, à l'aide d'un faisceau sorti sous balayage hélium (donc à la pression atmosphérique et sans prélèvement)

$-Z>9$ à $11(\mathrm{O}$ à $\mathrm{Na})$ 
- limite pratique de détection qq. $10^{-9}$, donc possibilité de mise en évidence de traces

- en outre, la réalisation d'un micro-faisceau extrait, d'un diamètre de l'ordre de $10 \mu \mathrm{m}$ et l'utilisation d'un système d'acquisition multiparamétrique et de déplacement pas à pas de l'échantillon sous le faisceau permet d'analyser de petits détails, tels que des inclusions dans les gemmes ou des enluminures, en effectuant des cartographies de concentration d'éléments. Applications : détermination de l'origine géographique de rubis sur une statue parthe $200 \mathrm{BC}$ du Louvre (Birmanie), détermination de la composition des pointes de métal utilisées pour le dessin par A. Dürer, Pisanello...

\subsubsection{RBS (Rutherford Backscattering)}

Détermination, à partir du spectre énergétique des protons rétrodiffusés de la teneur en différents constituants et / ou de l'épaisseur de couches superficielles.

Applications : détermination des profils de concentration élémentaire dans les couches de patine de bronzes, étude de l'altération de sceaux en plomb et contrôle des conditions de conservation de tels objets au moyen de coupons témoins en plomb.

\subsubsection{Réactions nucléaires}

Utilisation de réactions spécifiques, souvent à seuil, du type $(p, n),(p, 2 n),(d, n)$, etc...pour déterminer les teneurs en éléments légers dans des matrices métalliques.

Applications : détermination non destructive de la teneur en oxygène de bronzes archéologiques

2.5.4 Réalisation d'une ligne de faisceau expérimental, dédié à l'analyse par fluorescence $X$ des éléments légers, dite (PLXE) ${ }^{2}$

Si l'on veut réaliser l'analyse par fluorescence $X$ d'éléments légers présents dans une matrice constituée d'éléments lourds, une solution consiste à irradier par des particules chargées une cible primaire de numéro atomique intermédiaire entre ceux des éléments à doser et ceux de la matrice. La cible émet alors, selon le mode PIXE, des photons X qui permettent d'exciter à leur tour les raies caractéristiques des éléments légers à doser, sans être gêné par les éléments lourds majeurs de la matrice. Ainsi, une cible primaire de germanium produira des photons $\mathrm{X}_{\mathrm{K}}$ de $9,98 \mathrm{keV}$, énergie suffisante pour exciter les raies $X_{K}$ du cuivre et du zinc présents dans une matrice de plomb, sans interférer avec les raies $X_{L}$ de ce métal $(10,45$ et $10,55 \mathrm{keV})$ et donc de doser cuivre et zinc. 